This is the final peer-reviewed accepted manuscript of:

Francesco Benedetti, Paola Luches, Maria Chiara Spadaro, Gabriele Gasperi, Sergio D’Addato, Sergio Valeri, and Federico Boscherini, Structure and Morphology of Silver Nanoparticles on the (111) Surface of Cerium Oxide, in Journal of Physical Chemistry C , 2015, 119, 11, 6024-6032.

The final published version is available online at: https://doi.org/10.1021/jp5120527

Rights / License:

The terms and conditions for the reuse of this version of the manuscript are specified in the publishing policy. For all terms of use and more information see the publisher's website. 


\title{
Structure and Morphology of Ag Nanoparticles on the (111) Surface of Cerium Oxide
}

Francesco Benedetti ${ }^{1,2}$, Paola Luches ${ }^{2 *}$, Maria Chiara Spadaro ${ }^{1,2}$, Gabriele Gasperi ${ }^{1,2}$, Sergio D'Addato $^{1,2}$, Sergio Valeri ${ }^{1,2}$, Federico Boscherini ${ }^{3,4}$

${ }^{1}$ Dipartimento di Scienze Fisiche Informatiche e Matematiche, Università degli Studi di Modena e Reggio Emilia, Via G. Campi 213/a, 41125 Modena, Italy

${ }^{2}$ Consiglio Nazionale delle Ricerche, Istituto Nanoscienze, Via G. Campi 213/a, 41125 Modena, Italy

${ }^{3}$ Dipartimento di Fisica e Astronomia, Viale C. Berti Pichat 6/2, 40127 Bologna, Italy

${ }^{4}$ Istituto Officina dei Materiali, Consiglio Nazionale delle Ricerche, Operative Group in Grenoble, c/o ESRF, BP 220, F-38043 Grenoble, France

\begin{abstract}
The structure of Ag nanoparticles of different size, supported on the cerium oxide (111) surface, was investigated by X-ray absorption fine structure at the Ag K-edge. The results of the data analysis in the near and extended energy range are interpreted with the help of the results obtained by X-ray photoelectron spectroscopy and scanning tunneling microscopy measurements and allow to obtain a detailed atomic scale description of the model system investigated. The Ag nanoparticles have an average size of a few tens of $\AA$, which increases with increasing deposited Ag amount. The nanoparticles show a slight tendency to nucleate at the step edges between different cerium oxide layers and they have a face centered cubic structure with an Ag-Ag interatomic distance contracted by $3-4 \%$ with respect to the bulk value. The interatomic distance contraction is mainly ascribed to dimensionality induced effects, while epitaxial effects have a minor role. The presence of Ag-O bonds at the interface between the nanoparticles and the supporting oxide is also detected. The Ag-O interatomic distance decreases with decreasing nanoparticle size.
\end{abstract}

Keywords: Extended X-ray Absorption Fine Structure, Scanning Tunnelling Microscopy, X-ray Photoelectron Spectroscopy, dimensionality-induced lattice contraction, metal-oxide interface distance

* corresponding author: e-mail: paola.luches@unimore.it; telephone: +39-059-2055313; fax: +39059-2055235

\section{Introduction}


Cerium oxide is receiving a great interest in recent years, due to its high reducibility, i.e. due to the possibility for Ce ions to quickly modify their oxidation state in a reversible way. ${ }^{1}$ The reducibility of cerium oxide has been found to be enhanced by the proximity of metallic atoms in the form of dopants ${ }^{1-3}$ or by supported metallic nanoparticles (NPs). ${ }^{4}$ The $\mathrm{Ag} /$ cerium oxide system is gaining an increasing importance for catalysis, in particular for $\mathrm{CO}$ oxidation, ${ }^{5,6} \mathrm{NO}$ reduction, ${ }^{6}$ soot combustion in diesel engines antiparticulate filters, ${ }^{7-9}$ formaldehyde oxidation, ${ }^{10,11}$ and for its antibacterial activity. ${ }^{12}$ In most cases the $\mathrm{Ag} / \mathrm{CeO}_{2}$ catalyst showed a higher activity and durability than its individual components, and catalysts with lower $\mathrm{Ag}$ loadings, in which the $\mathrm{Ag}-\mathrm{CeO}_{2}$ interface has a larger relative weight, show lower activation temperatures. ${ }^{6}$ The strong interaction between $\mathrm{Ag}$ and cerium oxide ${ }^{9}$ has been shown to be largely dependent on the shape and stoichiometry of the oxide. ${ }^{5}$ Different active species for the chemical activity have been identified depending on the synthesis methods, which in turn determine the atomic scale details of the composite system. Shimitzu et al. give evidence for the formation of highly reducible surface oxygen at silver-ceria interface, and identify it as the active catalytic species. ${ }^{9}$ Aneggi et al. indicate a possible role of the $\mathrm{Ag}_{2} \mathrm{O}$ phase at the interface between metal particles and cerium oxide, ${ }^{8}$ while Machida et al. point at superoxo species formed on metallic silver particles as possible responsible for high catalytic activity. ${ }^{7}$

To fully understand and optimize the properties of the $\mathrm{Ag} / \mathrm{CeO}_{2}$ system, recent studies focused on model epitaxial systems, ${ }^{13-15}$ and on theoretical modeling, ${ }^{16-18}$ and evidenced a relevant interaction between the cerium oxide support and the metallic nanoparticles, ${ }^{13,14}$ the occurrence of oxygen reverse spillover from ceria to the $\mathrm{Ag}$ particles ${ }^{15}$ and a charge transfer from $\mathrm{Ag}$ to Ce cations. ${ }^{16} \mathrm{~A}$ better insight into the atomic scale details of the structure of the $\mathrm{Ag} \mathrm{NPs} / \mathrm{CeO}_{2}$ system is expected to be of help in obtaining a complete picture of the system, necessary to fully understand its properties. $\mathrm{X}$-ray absorption fine structure (XAFS) measurements in the near and extended energy range have proved to be very useful to have information on the structure and on the oxidation state of the cations in cerium oxide ${ }^{19-22}$ and of metallic NPs supported on cerium oxide, ${ }^{23-25}$ including also the $\mathrm{Ag} / \mathrm{CeO}_{2}$ system. ${ }^{9-26}$ However, previous studies addressed the structure of complex powdered systems, containing a wide variety of different NP/oxide interfaces. Furthermore the $\mathrm{Ag} / \mathrm{CeO}$ systems were prepared by chemical synthesis methods, which can also influence the interaction between nanoparticles and oxide supports. The use of model systems allows to select a specific cerium oxide surface, to accurately determine - and tune to some extent - the NP size distribution, to simultaneously focus on the intrinsic interactions between the metal and oxide surface under study, and to possibly obtain complementary information using surface science techniques. On the other hand, the application of the XAFS technique to model systems of NPs supported on a single oxide surface is quite challenging, due to the much smaller absolute number of absorbing atoms, compared to powdered systems.

The present study demonstrates that XAFS can be used to obtain refined information on the local structure of the $\mathrm{Ag} / \mathrm{CeO}_{2}$ model system for $\mathrm{Ag}$ particles of nanometric size. The information obtained by XAFS are complemented by a characterization using surface science techniques, such as X-ray photoemission spectroscopy (XPS) and scanning tunneling microscopy (STM) and give a detailed atomic scale description of the investigated system.

\section{Experimental details and data analysis}


The samples used for this study consist of submonolayer amounts of Ag deposited on cerium oxide films, in turn epitaxially grown on a Pt(111) substrate. The Ag atoms self assemble into NPs of increasing size as the deposited $\mathrm{Ag}$ amount is increased. ${ }^{16}$ The samples were prepared in an ultrahigh vacuum chamber (base pressure $1 \times 10^{-10} \mathrm{mbar}$ ), in which substrate preparation, film growth and in-situ XPS and STM analysis were performed. The Pt(111) substrate was prepared by cycles of sputtering $(1 \mathrm{KeV}, 1 \mu \mathrm{A})$ and annealing $(1040 \mathrm{~K})$, until the surface contamination was below the XPS detection limit. The cerium oxide films were prepared following the procedures exposed in Ref. 27 , i.e. evaporating $\mathrm{Ce}$ from an electron bombardment evaporation cell in oxygen partial pressure $\left(\mathrm{P}_{\mathrm{O}_{2}}=1 \times 10^{-7} \mathrm{mbar}\right)$, keeping the substrate at room temperature. To optimize the cerium oxide stoichiometry, the structural long range order and the lateral size and flatness of the cerium oxide terraces, the films were annealed at $770^{\circ} \mathrm{C}$ in $\mathrm{P}_{\mathrm{O}_{2}}=1 \times 10^{-7} \mathrm{mbar}$ for $15 \mathrm{~min}$ after the growth, following our previous study. ${ }^{27}$ The Ce evaporation cell was calibrated using a quartz crystal microbalance (Ce evaporation rate $\sim 0.2 \AA / \mathrm{min}$ ). In the following the cerium oxide film thickness will be expressed in monolayers (ML), defining the cerium oxide ML as the repeating unit along the [111] direction, i.e. one O-Ce-O trilayer with a height of $3.12 \AA$. The cerium oxide film thickness used in this study was $10 \mathrm{ML}$, chosen to have a closed film fully covering the Pt surface to avoid Ag/Pt interactions. Ag was grown by a Knudsen cell, calibrated by a quartz microbalance to have a rate of $0.4 \AA / \mathrm{min}$. The cerium oxide film was kept at room temperature during Ag growth. Four different submonolayer amounts of Ag are investigated in this study, namely $0.1 \AA, 0.2 \AA, 0.7 \AA$ and $1.5 \AA$.

XPS was used to determine the level of contaminants on the Pt surface during the cleaning procedure, to check the cerium oxide and Ag deposited amounts, and to investigate the oxidation state of Ag in the NPs after Ag deposition, before and after the XAFS measurements. XPS data were collected at normal take-off angle using an $\mathrm{Al} \mathrm{K}_{\alpha} \mathrm{X}$-ray source $(1486.6 \mathrm{eV})$ and a hemispherical electron analyzer. The spectra have been normalized in binding energy to have the substrate Pt $4 \mathrm{f}$ peak in the same position. The binding energy of Ag $3 \mathrm{~d}$ peaks was obtained by fitting the spectra with a Voigt-type lineshape.

STM was used to have information on the morphology of the Ag NPs. The STM images were acquired at RT in constant current mode, using electrochemically etched tungsten tips. The WSXM software was used for image processing. ${ }^{28}$

After growth and characterization by XPS and STM the samples were measured by XAFS at the Ag K-edge at the GILDA BM08 beamline, ${ }^{29}$ at the European Synchrotron Radiation Facility in Grenoble. The samples were kept in nitrogen atmosphere during the journey between the laboratory and the synchrotron, although sample mounting implied 15-30 minutes of air exposure. 
$\mathrm{V} 80^{\circ}$

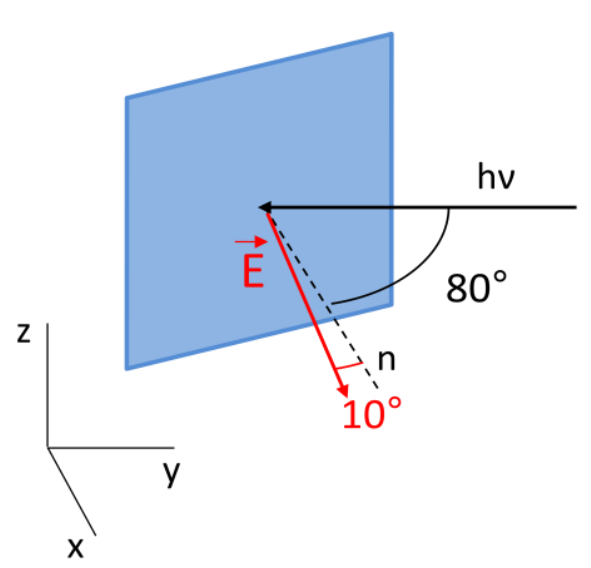

$\mathrm{V} 45^{\circ}$

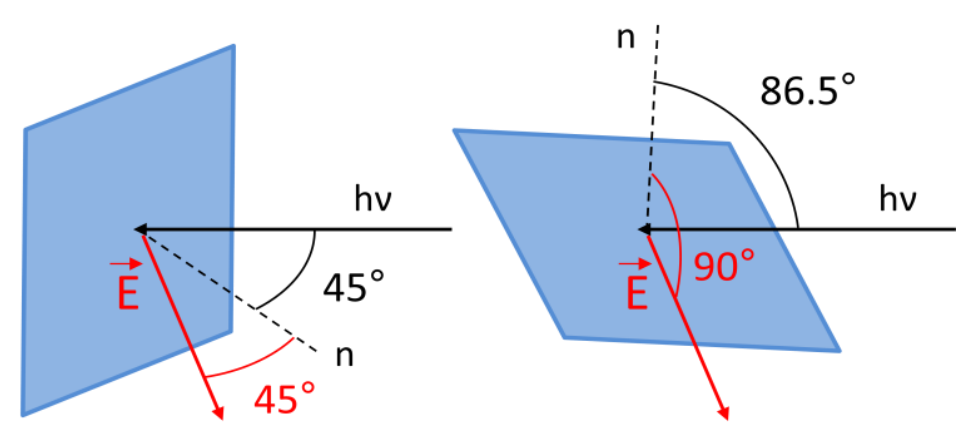

Figure 1: Sketch the experimental geometries used for the EXAFS measurements. V80 ${ }^{\circ}$ : sample in the vertical orientation and photon beam at $80^{\circ}$ from sample normal; $\mathrm{V} 45^{\circ}$ : sample in the vertical orientation and photon beam at $45^{\circ}$ from sample normal; $\mathrm{H} 86.5^{\circ}$ : sample in a nearly horizontal orientation and photon beam at $86.5^{\circ}$ from sample normal. The angles between photon polarization vector $\overline{\mathrm{E}}$ and sample normal $\mathrm{n}$ are also indicated in red.

The XAFS spectra were acquired in the fluorescence yield mode using a hyperpure Ge detector. In spite of the extremely low surface density of Ag atoms in our samples (ranging from $\sim 6$ to $90 \times 10^{13}$ atoms $/ \mathrm{cm}^{2}$ ), we could acquire spectra in the near energy range (X-ray absorption near edge structure, XANES) with an acceptable signal to noise ratio on all samples. The spectra in the extended energy range (X-ray absorption fine structure, EXAFS), instead, were acquired on two selected samples only, containing $0.2 \AA$ and $1.5 \AA \mathrm{Ag}$. On the sample with the largest amount of Ag we measured the EXAFS spectra in three different experimental geometries, i.e. with different relative orientation of the photon polarization vector and the sample normal, to selectively probe the NP structure in different orientations exploiting the polarization dependence of the X-ray absorption cross section. The different configurations used are shown in Fig.1: i) sample in the vertical orientation and photon beam at $80^{\circ}$ from sample normal $\left(\mathrm{V} 80^{\circ}\right)$; ii) same as i) with the photon beam at $45^{\circ}$ from sample normal $\left(\mathrm{V} 45^{\circ}\right)$; iii) sample in a nearly horizontal orientation and photon beam at $86.5^{\circ}$ from sample normal $\left(\mathrm{H} 86.5^{\circ}\right)$. In the H86.5 $5^{\circ}$ configuration the angle between the photon beam and the sample surface is close to the critical angle for total reflection from the Pt surface. In this configuration, in which a higher sensitivity to the surface layers is achieved, we measured the XANES spectra for all samples and the EXAFS spectrum for the $0.2 \AA \mathrm{Ag}$ sample. Two reference samples, namely a metallic Ag foil and an $\mathrm{Ag}_{2} \mathrm{O}$ sample in powdered form, were also measured in the transmission mode for comparison. Data processing and background subtraction were performed using the ATHENA software. ${ }^{30}$ Quantitative analysis was performed by fitting the EXAFS signals with the ARTEMIS package, ${ }^{31}$ using theoretical scattering functions simulated using the FEFF program. ${ }^{32}$ For each sample the ranges used for the fitting are $2-7 \AA^{-1}$ in $\mathrm{k}$ space and $1-3.5 \AA$ in $\mathrm{R}$ space. The fitting was performed simultaneously on $\mathrm{k}$-, $\mathrm{k}^{2}$ - and $\mathrm{k}^{3}$-weighted spectra. The same fitting ranges were used for the $\mathrm{Ag}$ and $\mathrm{Ag}_{2} \mathrm{O}$ reference sample spectra, to avoid systematic errors. 


\section{Results}

\subsection{Morphology}

STM was used to have information on the morphology of the bare surface of the $\mathrm{CeO}_{2}$ film and of the size shape and nucleation sites of the Ag NPs. Fig. 2 reports the STM images of the surface of the $10 \mathrm{ML}$ cerium oxide film before and after the deposition of $0.2 \AA$ and $1.5 \AA$ of $\mathrm{Ag}$. As observed in our previous works on $\mathrm{CeO}_{2} / \mathrm{Pt}(111)$ films, ${ }^{27,33}$ the bare cerium oxide film surface (Fig. 2a) exposes mainly two cerium oxide atomic layers separated by $\sim 3 \AA$, i.e. by one cerium oxide monolayer. The uppermost layer forms flat islands of several tens of $\mathrm{nm}$ lateral size. Both the cerium oxide islands and the underlying cerium oxide layer show some surface defects of nanometric size; the cerium oxide extended surface also shows some larger holes, which in turn expose the underlying cerium oxide layers, and some linear defects, ascribed to boundaries between twinned domains. ${ }^{27}$ An inspection of Fig.2b allows to clearly identify the Ag NPs which form after the deposition of $0.2 \AA$ Ag. The NPs show some tendency to nucleate at ceria step edges (some of them have been evidenced by yellow lines in Fig.2b), although many of them are also located on the cerium oxide terraces. A statistical analysis performed on several STM images allowed to estimate the fraction of NPs

a)

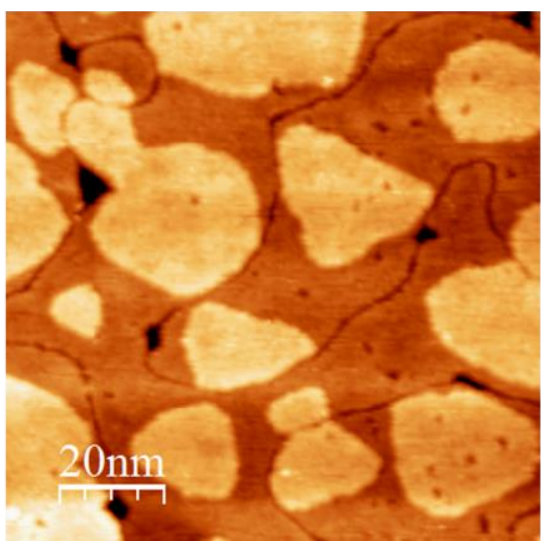

b)

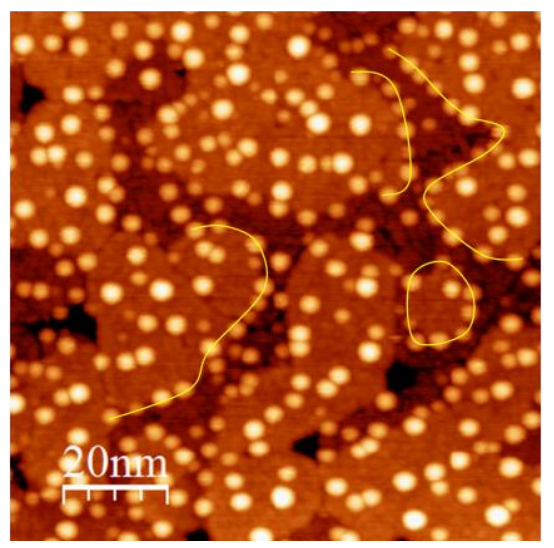

c)

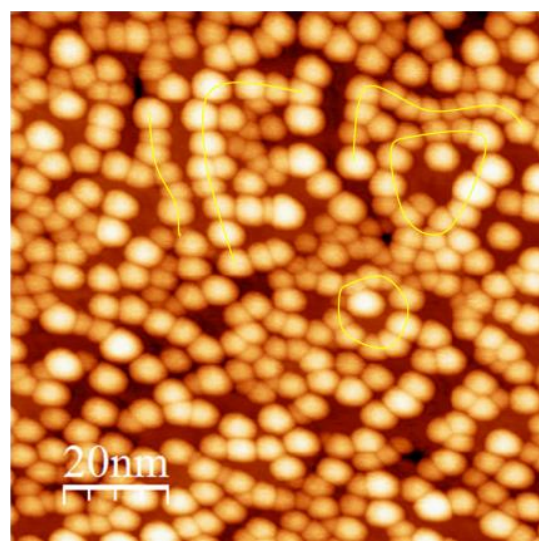

Figure 2: STM images of a $10 \mathrm{ML}$ cerium oxide film a) before, and after the deposition of b) $0.2 \AA \mathrm{Ag}$ and c) $1.5 \AA \mathrm{Ag}$. Measurement conditions: a) and c) V=3 V, I=0.03 nA; b) V=2.5 V, I=0.03 nA. The yellow lines in $b$ and $c$ evidence cerium oxide step edges on which Ag NP tend to nucleate.

nucleated at step edges as $64 \%$ of the total amount of NP formed. The size of the NPs is not uniform and it ranges from 3 to $17 \AA$ in height and from 20 to $40 \AA$ in lateral size. The average size of the NPs nucleated at the cerium oxide step edges is smaller than the average size of the NPs nucleated on the terraces. The average NP density, calculated on different $100 \times 100 \mathrm{~nm}^{2}$ images of the sample, is $2.8 \times 10^{12} \mathrm{NPs} / \mathrm{cm}^{2}$. Increasing the amount of deposited Ag to $1.5 \AA$ (Fig. $2 \mathrm{c}$ ) the NPs mainly increase in size, although their density is also slightly increased to $3.3 \times 10^{12} \mathrm{NPs} / \mathrm{cm}^{2}$. The NPs tend to be aligned along lines or closed paths (some of them are evidenced in Fig. 2c by yellow lines), recalling the shape of the cerium oxide island edges and indicating a preferential nucleation of the Ag NPs at ceria step edges also in this sample. The NP size in the $1.5 \AA \mathrm{Ag}$ sample ranges from 15 to $27 \AA$ in height and from 23 to $67 \AA$ in lateral size. In this sample it is more difficult to identify the preferential location of larger and smaller NPs. 


\subsection{Oxidation state of Ag NPs}

\subsubsection{XPS results}

To have an accurate description of the Ag NPs to model the XAFS data, we also investigated the Ag NP oxidation state before and after the XAFS measurements, since the latter were performed ex-situ and implied a short (approximately 15-30 minutes) air exposure during sample mounting. For this
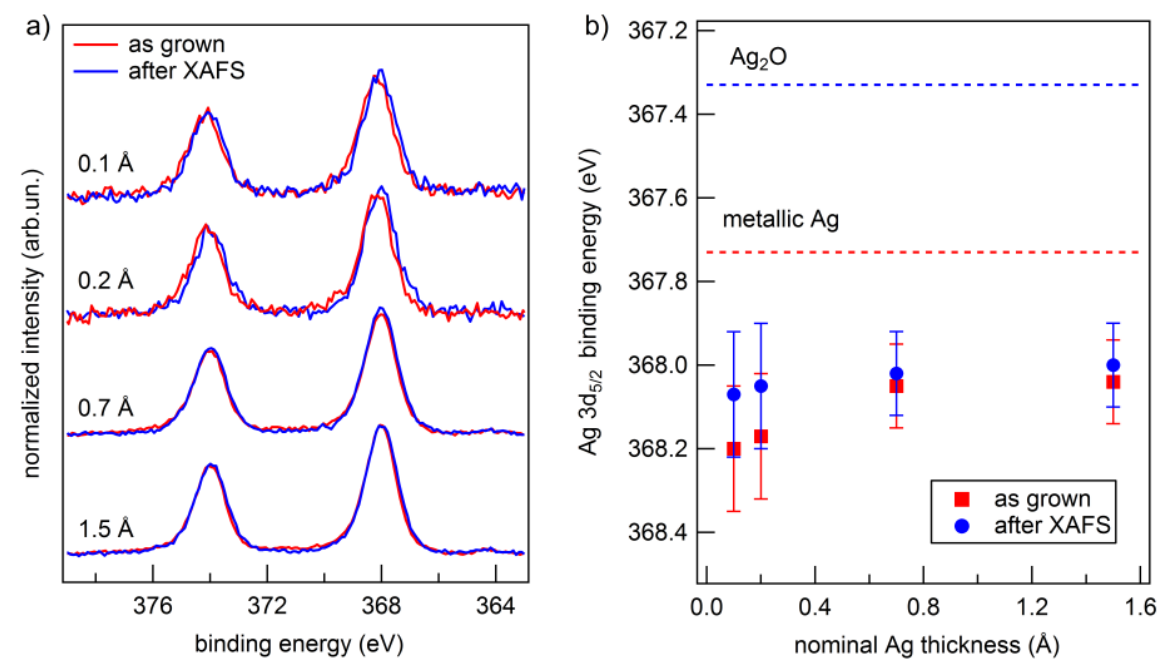

Figure 3: a) Ag 3d XPS spectra for samples with different Ag nominal thicknesses on a 10 ML cerium oxide film as grown (red lines) and after ex-situ XAFS measurements (blue lines). b) $\mathrm{Ag} 3 \mathrm{~d}_{5 / 2}$ binding energy obtained from the fitting of the spectra in a). The value measured on a metallic Ag $50 \AA$ film (taken from ref. (16)) and the value measured on an $\mathrm{Ag}_{2} \mathrm{O}$ sample (taken from ref. (41)) are also reported for comparison.

purpose we measured XPS Ag 3d spectra, shown in Fig.3a for the four different samples. The spectra in Fig.3a have been normalized in intensity to allow for a better comparison of the binding energy position and shape of the peaks. No relevant modification of the peak shape at the different Ag coverages was observed. Furthermore, the spectra after the XAFS measurements do not show any modification in width. Fig. 3 b reports the binding energy of the $\mathrm{Ag} 3 \mathrm{~d}_{5 / 2}$ peak for the different samples before and after XAFS measurements in comparison with the binding energy of a metallic $50 \AA$ thick $\mathrm{Ag}$ film on a cerium oxide film on $\mathrm{Pt}(111)$, already reported in Ref. 16, and the value for $\mathrm{Ag}_{2} \mathrm{O}$, which is expected to be $0.4 \mathrm{eV}$ shifted towards smaller binding energies. ${ }^{34,35}$ The binding energy of the $\mathrm{Ag}$ $3 \mathrm{~d}_{5 / 2}$ peaks in the NPs is larger than in metallic Ag, and in the as grown samples it increases with decreasing Ag coverage, i.e. NP size, due to dimensionality-related final state effects affecting the binding energy position. ${ }^{16}$ After air exposure the binding energy is slightly smaller. For the 1.5 and $0.7 \AA$ samples the shift is much smaller than the uncertainty of the measurement, while for the 0.2 and $0.1 \AA$ sample the shift amounts to $0.1 \mathrm{eV}$ and might indicate a slight surface oxidation after air exposure.

\subsubsection{XANES results}

Fig. 4 reports the Ag-K edge XANES spectra for the samples with different Ag nominal thickness and for the bulk metallic $\mathrm{Ag}$ and $\mathrm{Ag}_{2} \mathrm{O}$ reference samples. The spectrum of the $1.5 \AA \mathrm{Ag}$ sample is 


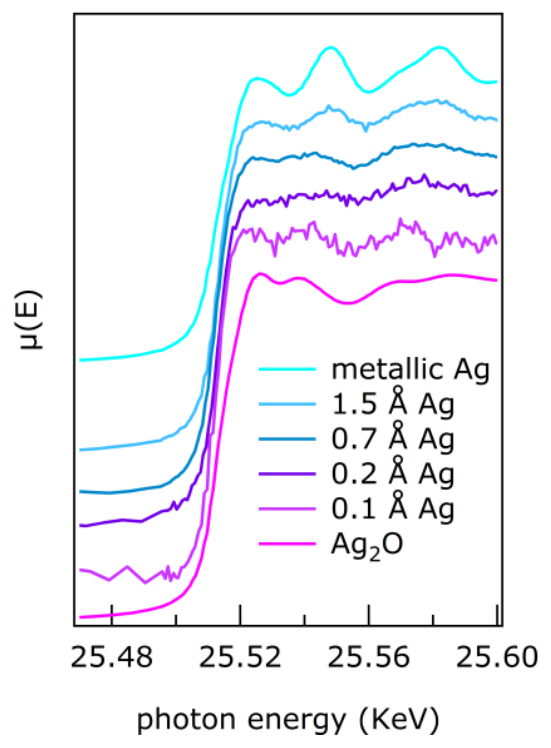

Figure 4: Ag K-edge XAFS spectra in the near edge energy range for the samples with different Ag nominal thickness on a $10 \mathrm{ML}$ cerium oxide film. The reference spectra of bulk metallic $\mathrm{Ag}$ and $\mathrm{Ag}_{2} \mathrm{O}$ are also shown for comparison.

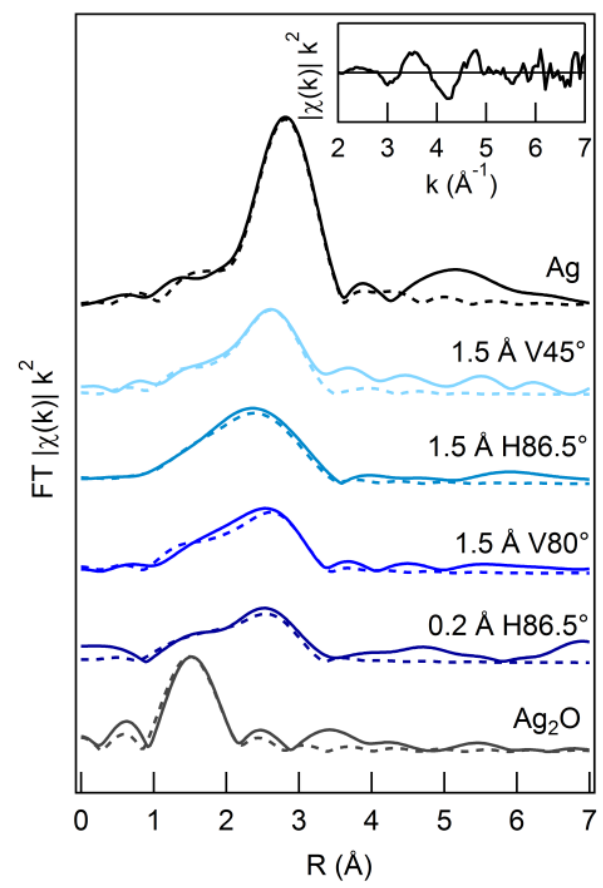

Figure 5: Modulus of the Fourier transform of the k2-weighted Ag K-edge $\chi(\mathrm{k})$ (solid lines) and fits (dashed lines) for the $1.5 \AA \mathrm{Ag}$ sample in three different experimental configurations (see fig.1), for the $0.2 \AA \mathrm{Ag}$ sample in the $\mathrm{H} 86.5^{\circ}$ configuration and for a metallic $\mathrm{Ag}$ and $\mathrm{Ag}_{2} \mathrm{O}$ reference samples. The inset reports the raw $\mathrm{k}^{2}$ weighted $\chi(\mathrm{k})$ data of the $1.5 \AA$ sample in the $\mathrm{V} 45^{\circ}$ configuration. NPs. very similar to the one of metallic Ag and it progressively evolves with decreasing $\mathrm{Ag}$ amount, becoming more similar to the $\mathrm{Ag}_{2} \mathrm{O}$ spectrum. Based on the results of the XPS analysis we can exclude significant surface oxidation of the Ag NPs. The evolution of the spectra shape with Ag thickness can be explained by an increasing relative weight of $\mathrm{Ag}$ atoms coordinated with oxygen atoms of the cerium oxide substrate as the $\mathrm{Ag}$ NPs become smaller as discussed in section 4 .

\subsection{Structure}

EXAFS spectra were measured to investigate the atomic scale structure of the Ag NPs. Due to the long times required for the acquisition of the spectra on the highly diluted samples, full EXAFS spectra were recorded in the three experimental geometries shown in Fig.1, only for the $1.5 \AA$ sample in order to detect possible structural anisotropies within the NPs. The $0.2 \AA$ sample was measured only in the $\mathrm{H} 86.5^{\circ}$ experimental geometry, to have a higher sensitivity to the Ag atoms which form the

Fig. 5 shows the magnitude of the Fourier transforms (FTs) of the $\mathrm{k}^{2}$ weighted $\chi(\mathrm{k})$ spectra of the $1.5 \AA \mathrm{Ag}$ sample, measured in the three experimental configurations (Fig. 1 ), of the $0.2 \AA$ sample in the $\mathrm{H} 86.5^{\circ}$ configuration, of a metallic $\mathrm{Ag}$ and of an $\mathrm{Ag}_{2} \mathrm{O}$ reference sample. The FT of the metallic Ag reference sample spectrum presents a dominant peak at $2.8 \AA$, which corresponds to first shell Ag-Ag scattering (Fig.5). On the contrary, the FT of the spectrum of the $\mathrm{Ag}_{2} \mathrm{O}$ reference sample is dominated by a peak at $1.5 \AA$, which corresponds to first shell Ag-O scattering (Fig.5). The FTs of the Ag NP samples show a rather broad peak with a maximum clearly shifted towards lower radial distances and with a lower intensity compared to the metallic $\mathrm{Ag}$ spectrum, and with a shoulder at low $\mathrm{R}$ values. The metallic Ag and $\mathrm{Ag}_{2} \mathrm{O}$ reference sample spectra were fit using $\mathrm{S}_{0}{ }^{2}$, the DebyeWaller factor $\sigma_{\mathrm{Ag}}{ }^{2}$ and the first shell interatomic distance $r$ as free parameters. The fitting gave values for these parameters in good agreement with the bulk values.

Fitting of the EXAFS data posed some peculiar problems due to the limited signal - to - noise ratio and the limited $\mathrm{k}-$ range. Moreover, any attempt at fitting of the spectra of the Ag NP samples using only the first shell Ag-Ag scattering paths, did not give satisfactory results. For this reason we introduced in the fitting also the Ag-O scattering path to account for the shoulder at low $\mathrm{R}$ values of the main peak. 
Since $\mathrm{Ag}$ atoms are present in two environments (bonded to $\mathrm{Ag}$ and to $\mathrm{O}$ ) it must be stressed that it is impossible to independently determine the coordination numbers and the relative fraction of $\mathrm{Ag}$ atoms in the two local coordinations; hence, in the following, the coordination numbers were assumed to be equal to the bulk values, a clear but inevitable limitation of our approach. The model used for the fitting includes a fraction $\beta$ of Ag atoms in the NP in a metallic phase, i.e. coordinated only with $\mathrm{Ag}$ atoms, and a fraction 1- $\beta$ of $\mathrm{Ag}$ atoms with $\mathrm{O}$ coordination. We chose to limit as much as possible the number of free parameters to be used in the fitting of the NP spectra. Therefore, we first performed a fit in the 2.0 - 3.5 $\AA \mathrm{R}$ range including only the $\mathrm{Ag}$ - $\mathrm{Ag}$ first coordination shell scattering path. In this first step $\mathrm{S}_{0}{ }^{2}$ and the $\mathrm{Ag}-\mathrm{Ag}$ coordination number $(\mathrm{CN})$ were fixed to the bulk value $\left(\mathrm{S}_{0}{ }^{2}=0.9\right.$ and $\mathrm{CN}=12$ ), while the Ag-Ag interatomic distance, the Debye-Waller (DW) factor, the $\mathrm{E}_{0}$ value and the weight $\beta$ were left as free parameters. The second step consisted in extending the $\mathrm{R}$ fitting range to $1.0-3.5 \AA$, fixing the $\beta$ parameter to the value obtained in the first fitting step, and introducing the Ag-O scattering path with a fixed weight 1- $\beta$, a fixed DW factor $0.002 \AA^{2}$ (obtained from bulk $\operatorname{Ag}_{2} \mathrm{O}$ ) and a free Ag-O distance. The Ag-Ag DW factor for the final fitting was fixed to the value obtained from the fitting of the $1.5 \AA \mathrm{V} 45^{\circ}$ sample spectrum $\left(0.023 \AA^{2}\right)$, which showed the best signal-to-noise ratio. Using fixed DW factors is clearly a crude approximation, but it was found to be the only way to obtain a reasonably good fit; note that all our following discussion is based on the variation of interatomic distances, thus no particular significance is given to the parameters affecting the amplitude of the signal such as $\beta$ and the DW factors. The parameters obtained from the fitting of all the EXAFS spectra measured on the Ag NP samples are reported in Tab.1. Alternative fitting procedures including different approximations gave very similar results in terms of Ag-Ag and AgO interatomic distances (see supporting information).

Table 1: Parameters obtained from the fitting of the EXAFS spectra ${ }^{\mathrm{a}}$

\begin{tabular}{|c|c|c|c|}
\hline sample & $\mathbf{R}_{\mathbf{A g}-\mathbf{A g}}(\mathbf{\AA})$ & $\mathbf{R}_{\mathrm{Ag}-\mathbf{O}}(\mathbf{A})$ & $\boldsymbol{\beta}$ \\
\hline $\mathbf{A g}$ & $\begin{array}{c}2.912 \pm \\
0.003\end{array}$ & - & 1 \\
\hline $\mathbf{A g}_{\mathbf{2}} \mathbf{O}$ & - & $2.06 \pm 0.03$ & 0 \\
\hline $\mathbf{1 . 5} \mathbf{\mathbf { ~ } \mathbf { V 4 5 } ^ { \circ }}$ & $2.82 \pm 0.02$ & $2.11 \pm 0.03$ & 0.8 \\
\hline $\mathbf{1 . 5} \AA \mathbf{~ V 8 0}^{\circ}$ & $2.78 \pm 0.07$ & $2.17 \pm 0.03$ & 0.4 \\
\hline $\mathbf{1 . 5} \AA \mathbf{H 8 6 . 5}^{\circ}$ & $2.79 \pm 0.06$ & $2.19 \pm 0.03$ & 0.6 \\
\hline $\mathbf{0 . 2} \AA \mathbf{H 8 6 . 5}^{\circ}$ & $2.78 \pm 0.06$ & $2.12 \pm 0.04$ & 0.3 \\
\hline
\end{tabular}

a $\mathrm{Ag}-\mathrm{Ag}$ nearest neighbour distance $\left(\mathrm{R}_{\mathrm{Ag}-\mathrm{Ag}}\right), \mathrm{Ag}-\mathrm{O}$ nearest neighbour distance $\left(\mathrm{R}_{\mathrm{Ag}-\mathrm{O}}\right)$, fraction of atoms in the metallic phase $(\beta)$.

The Ag-Ag nearest neighbor distance in the NPs is significantly contracted by 3-4 \% with respect to the bulk metallic phase. The different experimental geometries for the $1.5 \AA \mathrm{Ag}$ sample give comparable values for the interatomic distance contraction, indicating the absence of evident anisotropies in the NPs structure. The Ag-Ag distance contraction in the $0.2 \AA$ and $1.5 \AA \mathrm{Ag}$ samples in the $\mathrm{H} 86.5^{\circ}$ configuration are very similar and indicate that within the investigated NP size range the contraction is comparable. The Ag-O scattering distances in the NP samples are larger than in 
$\mathrm{Ag}_{2} \mathrm{O}$ by $3-6 \%$. The Ag-O distance in the $0.2 \AA \mathrm{Ag}$ sample in the $\mathrm{H} 86.5^{\circ}$ configuration is shorter than in the $1.5 \AA \mathrm{Ag}$ sample in the same experimental configuration. The fraction of $\mathrm{Ag}$ atoms in the metallic phase in the $1.5 \AA \mathrm{Ag}$ sample measured in the $\mathrm{H} 86.5^{\circ}$ configuration is larger than in the 0.2 $\AA$ Ag sample in the same experimental geometry, consistent with the decrease of average NP size with decreasing Ag nominal thickness as discussed in section 4.

\section{Discussion}

The aim of this work was to use EXAFS to have atomic scale information on the structure of Ag NPs and of the interface with $\mathrm{CeO}_{2}$, using a well controlled model epitaxial system. First of all we point out that the extreme dilution of the samples have made the EXAFS measurements rather challenging. Nevertheless, we showed that it is indeed possible to obtain valuable information on the structure of this system by EXAFS, combined with surface science techniques, such as STM and XPS.

It was known from previous work that $\mathrm{Ag}$ deposited on $\mathrm{CeO}_{2}(111)$ surfaces spontaneously forms NPs, which increase their size with increasing amount of deposited Ag. ${ }^{13,15,16}$ However existing studies mainly focus on the interaction between $\mathrm{Ag}$ and $\mathrm{CeO}_{2}$ rather to the detailed morphology of the system. The STM images of Fig. 2 show that the Ag NPs show a slight tendency for nucleation at cerium oxide step edges. A similar behaviour was already observed for Rh and Pd and Au NPs on stoichiometric ceria films, ${ }^{36,37}$ while the absence of preferential sites was observed for Au and Pd NPs on partially reduced cerium oxide surfaces with a high density of surface defects. ${ }^{37-39}$ A statistical analysis of our images indicates that $64 \%$ of the NP are nucleated at the step edges. However this number has to be related to the density of step edges of the substrate. ${ }^{36}$ Zhou et al. measured a comparable fraction of Rh NPs nucleated at cerium oxide step edges, although in their substrates show a significantly smaller density of step edge than the ones used in the present study. ${ }^{36}$ So, certainly the tendency for Ag NPs to nucleate at step edges is not as relevant as for Rh NPs. Nevertheless the significant fraction of NPs nucleated at step edges also on our samples indicates that, in spite of the charge transfer and the strong interaction occurring at the interface for this system, ${ }^{14,16}$ the $\mathrm{Ag}$ atoms have some mobility on the $\mathrm{CeO}_{2}(111)$ surface. The larger average size of the NPs on the terraces compared to the NPs nucleated at the step edges is due to the larger mobility of Ag atoms on the flat surface. The NP size and densities observed in this work are very similar to the ones observed on a 3 ML thick cerium oxide film which has a similar density of step edges. ${ }^{16}$ Slightly different values of NP density obtained in previous works on the $\mathrm{Ag} / \mathrm{CeO}_{2}$ model system ${ }^{13,15}$ confirm that the actual NP density depends on the step and defect densities, obtained for the different procedures used for cerium oxide film growth. The size of metallic nanoparticles on oxide surfaces, as measured by STM, cannot be considered fully reliable. In particular, the lateral size is overestimated by the convolution with the finite tip size. The NP heights can also be possibly affected by the different density of states of the oxide film and the metal NPs depending on the tunneling bias used for the measurement. ${ }^{40,41}$ The thickness of cerium oxide films has been indeed found to be dependent on the tunneling bias for thin samples. ${ }^{27}$ However, for the $10 \mathrm{ML}$ films here investigated the image quality was not satisfactory at tunneling biases lower than $2.5 \mathrm{~V}$. We can assume that a 10 ML cerium oxide film has a band gap close to the bulk oxide, $6 \mathrm{eV}$, and that the sample-to-tip voltages used in this study - from 2.5 to $3.5 \mathrm{~V}$ - probe the states in the conduction band of cerium oxide, since no significant variations in the particle height were observed by changing the tunneling bias from 2.5 $\mathrm{V}$ to $3.5 \mathrm{~V}$. For this reason we can consider the correction to the measured particles height distribution 
to be rather small. The average NP height is $10 \AA$ in the $0.2 \AA \mathrm{Ag}$ sample and $20 \AA$ in the $1.5 \AA \mathrm{Ag}$ sample. The equilibrium shape of the Ag NPs, at least for the largest NPs, is expected to be determined by the modified Wulff construction, ${ }^{42}$ giving a truncated-octahedral shape with the (111) orientation which can be approximated by a hemisphere with a lateral size approximately twice the height, as deduced also from previous works on the same system. ${ }^{13-15}$ This hypothesis is in agreement with an overestimate of the average lateral dimensions by STM. Given the limited size of the NPs and the preferential step edge nucleation, a significant fraction of the Ag atoms of the Ag NPs are expected to be coordinated with $\mathrm{O}$ atoms, since the cerium oxide (111) terraces ideally terminate with a plane of $\mathrm{O}$ atoms and oxygen atoms are also exposed along the cerium oxide step edges.

Another interesting point to discuss is the effect of air exposure on the Ag NPs. Ag NPs with size comparable to the ones here investigated, have been found to have a tendency to be oxidized by air exposure, which induces a shift of the binding energy of XPS Ag 3d peaks of $0.4 \mathrm{eV}$ or larger. ${ }^{43,44}$ Cai et al. observed a $0.7 \mathrm{eV}$ shift in Ag $3 \mathrm{~d}$ binding energy of $30 \AA$ diameter Ag NPs on HOPG after air exposure and ascribe it to the formation of a dense $\mathrm{Ag}_{2} \mathrm{O}$ layer on the NP surface. ${ }^{43}$ Schnippering et al. instead observe a $0.4 \mathrm{eV}$ negative binding energy shift in Ag NP when the diameter is decreased from 70 to $40 \AA$ due to a partial surface oxidation. ${ }^{44}$ The samples here investigated show a negative binding energy shift of Ag 3d XPS peaks after air exposure. For 1.5 and $0.7 \AA \mathrm{Ag}$, however, the shift is negligible, while for the 0.2 and $0.1 \AA$ samples it is significantly smaller than the ones observed in the literature. Therefore, although we cannot exclude a slight surface oxidation, it is certainly not significant. This is possibly related to the specific Ag cerium oxide system, in which the charge transfer occurring between cerium oxide and the $\mathrm{Ag} \mathrm{NPs}{ }^{16}$ may partially inhibit further Ag oxidation induced by oxygen molecules contained in the atmosphere.

On the other hand, some modification of the Ag XANES was observed with decreasing NP size (Fig.4), consistent with a modification of the density of empty states which becomes progressively closer to the one of $\mathrm{Ag}_{2} \mathrm{O}$. Furthermore, Ag-O coordinations were found to influence the EXAFS spectra (Fig.5). The charge transfer from the Ag NPs towards the cerium oxide substrate, ${ }^{16}$ with the oxidation of interface Ag atoms, can be responsible for the observed modification of the XANES spectra in the smallest NPs, in which the relative weight of interface atoms is certainly larger than in the bigger ones. Moreover, the contribution of surface Ag-O bonds formed by air exposure of the samples cannot be excluded. However, the Ag-O interatomic distance observed by EXAFS (Tab. 1) is expanded with respect to the value in $\mathrm{Ag}_{2} \mathrm{O}$, further supporting the idea that the NPs are not significantly oxidized and that a relevant contribution to Ag-O scattering comes from $\mathrm{O}$ atoms in the cerium oxide support. Individual Ag atoms, adsorbed on the hollow site of the cerium oxide (111) surface, i.e. on top of the $\mathrm{O}$ atoms in the third $\mathrm{O}$ layer below the surface (most stable configuration) have an Ag-O distance of $2.27 \AA .{ }^{17,18}$ Although a calculation for NPs with a size comparable to the ones here investigated is not available, the average distance calculated for an Ag single layer on the cerium oxide (111) surface, $2.44 \AA$, and the shortest Ag-O distance for the same system, $2.19 \AA$, ${ }^{16}$ both expanded with respect to $\mathrm{Ag}_{2} \mathrm{O}$, are in an at least qualitative agreement with the value obtained by EXAFS. In an EXAFS study of a combustion-synthesized $\mathrm{Ag} / \mathrm{CeO}_{2}$ catalyst a similar Ag-O distance $(2.19 \AA)$ has been observed and ascribed to $\mathrm{Ag}^{+}$ions coordinated with oxygen in the support. ${ }^{26}$ These evidences, combined with the results of XPS, further support the hypothesis that a relevant contribution to the measured $\mathrm{Ag}-\mathrm{O}$ comes from scattering at the interface with the support. At lower Ag coverages the Ag-O distance measured in the same experimental configuration 
decreases, possibly as a consequence of the stronger interaction between Ag and cerium oxide in smaller NPs, due to the larger charge transfer per atom in NPs with a smaller size. ${ }^{16}$

The analysis of the EXAFS spectra pointed out a contraction of the nearest neighbor Ag-Ag distance, which is possibly due to dimensionality-related effects. As pointed out by Kern and Müller, ${ }^{45}$ in general, supported NPs undergo an active misfit $\mathrm{m}$, given by the sum of a contribution related to epitaxy and a contribution related to reduced dimensionality: $m=m_{0}+\Delta a / a$, where $m_{0}$ is the epitaxial mismatch and $\Delta \mathrm{a} / \mathrm{a}$ is the contraction related to reduced dimensionality. Following a study by Lagarde et al. on $\mathrm{Ag}$ NPs on $\mathrm{MgO}(111),{ }^{46}$ we calculated the different contributions for the $\mathrm{Ag} / \mathrm{CeO}_{2}(111)$ case. The epitaxial relation is (111) $\mathrm{Ag} / /(111) \mathrm{CeO}_{2}$ and [110] $\mathrm{Ag} / /[110] \mathrm{CeO}_{2}(16)$. The $\mathrm{Ag}$ and $\mathrm{CeO}_{2}$ lattice parameters on the (111) surface, $2.889 \AA$ and $3.826 \AA$ respectively, are quite different and the epitaxy occurs via the formation of a supercell with four $\mathrm{Ag}$ cells superimposed on three $\mathrm{CeO}_{2}$ cells. ${ }^{16}$ In this case the $\mathrm{Ag} / \mathrm{CeO}_{2}$ epitaxial mismatch is $m 0=\frac{4 a_{\mathrm{Ag}}-3 a_{\mathrm{CeO}}}{4 a_{\mathrm{Ag}}}=0.7 \%$. In Fig.6 we reported the Ag-Ag first shell interatomic distances for bulk metallic $\mathrm{Ag}$, the value of the lattice parameter expected for an epitaxial contraction and the value measured by EXAFS for the $0.2 \AA$ and $1.5 \AA$ samples in the $\mathrm{H} 86.5^{\circ}$ geometry. The contraction observed in the NP samples is clearly much larger than the epitaxial contraction. In a macroscopic description ("liquid drop" model) ${ }^{47}$ dimensionality-related lattice contraction in spherical NPs with cubic structure and radius $r$ can be related to surface stress $f$ and to bulk compressibility $\kappa$ by:

Figure 6: $\mathrm{Ag}-\mathrm{Ag}$ interatomic distance for the $0.2 \AA \mathrm{Ag}$ and $1.5 \AA \mathrm{Ag}$ samples on $\mathrm{CeO}_{2}$ measured by EXAFS in the $\mathrm{H} 86.5^{\circ}$ configuration (red circles). The thick grey line is the value measured on the metallic reference sample (the line thickness is proportional to the error bar). The green line is the value expected assuming an epitaxial contraction of $0.7 \%$. The black squares represent the values obtained for the dimensionality-related contraction using the "liquid drop" model.

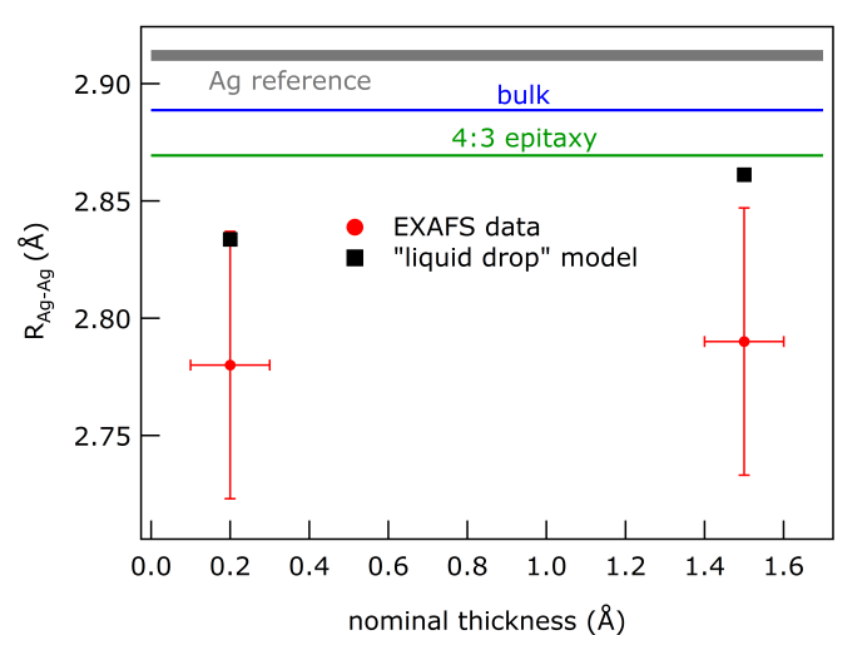

$$
\frac{\Delta \mathrm{a}}{\mathrm{a}}=-\frac{2}{3} \kappa\left(\frac{f}{r}\right)
$$

Using the bulk compressibility of silver $\kappa=10 \times 10^{-12} \mathrm{~m}^{2} / \mathrm{N}, \mathrm{f}=2.286$ $\mathrm{N} / \mathrm{m},{ }^{48}$ and for $\mathrm{r}$ the average values measured by STM for the height of the NPs, i.e. $10 \AA$ for the $0.2 \AA \mathrm{Ag}$ sample and $20 \AA$ for the $1.5 \AA \mathrm{Ag}$ sample, we obtain a $1.9 \%$ and a 0.95 $\%$ contraction in the $0.2 \AA$ and $1.5 \AA$ Ag samples respectively. Fig. 6 shows that the Ag-Ag distances obtained in this way are larger than the values measured by EXAFS on the two investigated samples. This discrepancy can be explained considering the approximations under which (1) is valid and the uncertainties in the parameters included in it. Equation (1) is based on a macroscopic relation between surface stress and hydrostatic pressure in a spherical nanoparticle of radius $r$. It has been shown to be valid also in the limit of particles with diameter of a few tens of $\AA, 48-50$ however at the very small sizes here investigated some deviations can certainly occur. Furthermore, the supported NPs here investigated are not spherical and their size in each sample is not uniform. A wide range of values for Ag surface stress $f$ can be found in the literature and we have used a value determined experimentally 
on Ag NPs, ${ }^{48}$ which was considered to be the system which better approximated our samples, though the NP size was larger size than in the system here investigated. However, even under these approximations, the model gives an expected dimensionality-induced contraction which is larger than the contraction which would be induced by epitaxial mismatch and we believe that this is the major origin of the observed contraction. This hypothesis is substantiated by the isotropy of the contraction observed by EXAFS in the three experimental geometries for the $1.5 \AA$ film (Tab.1). Some anisotropy between the in-plane and out-of-plane contractions would in fact be expected in the case of an epitaxial effect.

An interatomic distance smaller than the bulk phase in metallic particles with nanometric size was observed by EXAFS since the late seventies ${ }^{48-51}$ and explained by the reduction of repulsive interactions between electrons which do not contribute to the bonding, given the lower average atomic coordination number in low dimensional systems. The bond length contraction, and the modifications of electronic structure at the nanoscale, are responsible for a number of new and different physical and chemical properties in nanoparticles. ${ }^{52}$ In particular for Ag NPs different optical ${ }^{52,53}$ and electrochemical properties ${ }^{54}$ have been observed in recent years. The interatomic distance contraction observed in the Ag NPs here investigated is comparable with the ones measured for Ag NP systems of similar size within more complex systems ${ }^{46,48}$ and it is expected to be relevant for a correct description of the system, which is necessary to understand the properties of $\mathrm{Ag} \mathrm{NPs}$ on $\mathrm{CeO}_{2}$.

\section{Conclusions}

This work shows that Ag NPs of nanometric size supported on the $\mathrm{CeO}_{2}(111)$ surface have the fcc structure with a 3-4\% contraction in the interatomic distance. The Ag-O interface distance decreases with decreasing NP size, possibly due to a larger charge transfer per Ag atom between the NPs and cerium oxide. The interaction between the Ag NPs and the $\mathrm{O}$ atoms of the cerium oxide support induces a modification of the empty states structure, which is progressively more evident as the NP size decreases. The observed resistance of the NPs towards oxidation after a short air exposure could be due to the charge transfer between the Ag and the oxide.

A complete identification of the atomic scale structure of metal NPs supported on oxides is very important in this and in other combined metal / oxide systems, in which the atomic scale details determine the material properties. This work shows that using EXAFS at the Ag K-edge it is possible have the required information even for subnanometric amounts of deposited $\mathrm{Ag}$ on a cerium oxide single crystal oxide surfaces.

\section{Acknowledgements}

This work was supported by the Italian MIUR through the FIRB Project RBAP115AYN "Oxides at the nanoscale: multifunctionality and applications" and by the COST Action CM1104 "Reducible oxide chemistry, structure and functions". We are grateful for the support of the staff of the GILDA beamline at ESRF, in particular F. d'Acapito and S. Torrengo.

\section{References}

(1) Trovarelli, A.; Fornasiero, P. Catalysis by Ceria and Related Materials; London: Imperial College Press, 2013. 
(2) Vecchietti, J.; Collins, S.; Xu, W.; Barrio, L.; Stacchiola, D.; Calatayud, M.; Tielens, F.;

Delgado, J. J.; Bonivardi, A. Surface Reduction Mechanism of Cerium-Gallium Mixed Oxides with Enhanced Redox Properties. J. Phys. Chem. C 2013, 117, 8822-8831.

(3) Shapovalov, V.; Metiu, H. Catalysis by Doped Oxides: CO Oxidation by $\mathrm{Au}_{x} \mathrm{Ce} 1-x \mathrm{O} 2$. J. Catal. 2005, 245, 205-214.

(4) Vayssilov, G. N.; Lykhach, Y.; Migani, A.; Staudt, T.; Petrova, G. P.; Tsud, N.; Skala, T.; Bruix, A.; Illas, F.; Prince, K. C.; Matolin, V.; Neyman, K. M.; Libuda, J. Support Nanostructure Boosts Oxygen Transfer to Catalytically Active Platinum Nanoparticles. Nat. Mat. 2011, 10, 310315.

(5) Chang, S.; Li, M.; Hua, Q.; Zhang, L.; Ma, Y.; Ye, B.; Huang, W. Shape-Dependent Interplay Between Oxygen Vacancies and $\mathrm{Ag}-\mathrm{CeO}_{2}$ Interaction in $\mathrm{Ag} / \mathrm{CeO}_{2}$ Catalysts and Their Influence on the Catalytic Activity. J. Catal. 2012, 293, 195-204.

(6) Bera, P.; Patil, K. C.; Hegde, M. S. NO Reduction, CO and Hydrocarbon Oxidation Over Combustion Synthesized $\mathrm{Ag} / \mathrm{CeO}_{2}$ Catalyst. Phys. Chem. Chem. Phys. 2000, 2, 3715-3719.

(7) Machida, M.; Murata, Y.; Kishikawa, K.; Zhang, D.; Ikeue, K. On the Reasons for High Activity of $\mathrm{CeO}_{2}$ Catalyst for Soot Oxidation. Chem. Mater. 2008, 20, 4489-4494.

(8) Aneggi, E.; Llorca, J.; de Leitenburg, C.; Dolcetti, G.; Trovarelli, A. Soot Combustion Over Silver-Supported Catalysts. Appl. Cat. B 2009, 91, 489-498.

(9) Shimizu, K.; Kawachi, H.; Satsuma, A. Study of Active Sites and Mechanism for Soot Oxidation by Silver-Loaded Ceria Catalyst. Appl. Cat. B 2010, 96, 169-175.

(10) Imamura, S.; Uchihori, D.; Utani, K.; Ito, T. Oxidative Decomposition of Formaldehyde on Silver-Cerium Composite Oxide Catalyst. Catal. Lett. 1994, 24, 377-384.

(11) Ma, L.; Wang, D.; Li, J.; Bai, B.; Fu, L.; Li. Y. Ag/CeO 2 Nanospheres: Efficient Catalysts for Formaldehyde Oxidation. Appl. Cat. B 2014, 148-149, 36-43.

(12) Wang, L.; He, H.; Yu, Y.; Sun, L.; Liu, S.; Zhang C.; He, L. Morphology-Dependent Bactericidal Activities of $\mathrm{Ag} / \mathrm{CeO}_{2}$ Catalysts Against Escherichia Coli. J. In. Biochem. 2014, 135, 45-53.

(13) Farmer, J. A.; Baricuatro, J. H.; Campbell, C. T. Ag Adsorption on Reduced $\mathrm{CeO}_{2}(111)$ Thin Films. J. Phys. Chem. C 2010, 114, 17166-17172.

(14) Farmer, J. A.; Campbell, C. T. Ceria Maintains Smaller Metal Catalyst Particles by Strong Metal-Support Bonding. Science. 2010, 329, 933-936.

(15) Kong, D. ; Wang, G.; Pan, Y.; Hu, S.; Hou, J.; Pan, H.; Campbell C. T.; Zhu, J. Growth, Structure, and Stability of Ag on CeO2(111): Synchrotron Radiation Photoemission Studies. J. Phys. Chem. C 2011, 115, 6715-6725. 
(16) Luches, P. ; Pagliuca, F.; Valeri, S.; Illas, F.; Preda, G.; Pacchioni, G. Nature of Ag Islands and Nanoparticles on the CeO2(111) Surface. J. Phys. Chem C 2012, 116, 1122-1132.

(17) Branda, M.; Hernandez, N.; Sanz, J.; Illas, F. Density Functional Theory Study of the Interaction of $\mathrm{Cu}, \mathrm{Ag}$, and $\mathrm{Au}$ Atoms with the Regular $\mathrm{CeO}_{2}$ (111) Surface. J. Phys. Chem. C 2010, 114, 1934-1941.

(18) Preda, G.; Pacchioni, G. Formation of Oxygen Active Species in Ag-Modified $\mathrm{CeO}_{2} \mathrm{Catalyst}$ for Soot Oxidation: A DFT study. Cat. Tod. 2011, 177, 31-38.

(19) Bianconi, A.; Marcelli, A.; Dexpert, H.; Karnatak, R.; Kotani, A.; Jo, T.; Petiau, J. Specific Intermediate-Valence State of Insulating $4 f$ Compounds Detected by $\mathrm{L}_{3} \mathrm{X}$-Ray Absorption. Phys. Rev. B 1987, 35, 806-812.

(20) Soldatov, A. V.; Ivanchenko, T. S.; Della Longa, S.; Kotani, A.; Iwamoto, Y.; Bianconi, A. Crystal-Structure Effects in the $\mathrm{Ce} \mathrm{L}_{3}$-Edge X-Ray-Absorption Spectrum of $\mathrm{CeO}_{2}$ : MultipleScattering resonances and many-body final states. Phys. Rev. B 1994, 50, 5074-5080.

(21) Vlaic, G.; Di Monte, R.; Fornasiero, P.; Fonda, E.; Kaspar, J.; Graziani, M. Redox PropertyLocal Structure Relationships in the Rh-Loaded $\mathrm{CeO}_{2}-\mathrm{ZrO}_{2}$ Mixed Oxides. J. Catal. 1999, 182, 378-389.

(22) Luches, P.; Pagliuca, F.; Valeri, S.; Boscherini, F. Structure of Ultrathin $\mathrm{CeO}_{2}$ Films on $\mathrm{Pt}(111)$ by Polarization-Dependent $\mathrm{X}$-ray Absorption Fine Structure. J. Phys. Chem. C 2013, 117, 1030-1036.

(23) Longo, A. ; Liotta, L. F.; Pantaleo, G.; Giannici, F.; Venezia, A. M.; Martorana, A. Structure of the Metal-Support Interface and Oxidation State of Gold Nanoparticles Supported on Ceria. $J$. Phys. Chem. C 2011, 116, p. 2960-2966.

(24) Gamarra, D.; Munuera, G.; Hungria, A. B.; Fernandez-Garcia, M.; Conesa, J. C.; Midgley, P. A.; Wang, X. Q.; Hanson, J. C.; Rodriguez, J. A.; Martinez-Arias, A. Structure-Activity Relationship in Nanostructured Copper-Ceria-Based Preferential CO Oxidation Catalysts. J. Phys. Chem C 2007, 111, 11026-11038.

(25) Si, R.; Tao, J.; Evans, J.; Park, J. B.; Barrio, L.; Hanson, J. C.; Zhu, Y. M.; Hrbek, J.; Rodriguez, J. A. Effect of Ceria on Gold-Titania Catalysts for the Water-Gas Shift Reaction: Fundamental Studies for $\mathrm{Au} / \mathrm{CeO}_{\mathrm{x}} / \mathrm{TiO}_{2}(110)$ and $\mathrm{Au} / \mathrm{CeO}_{\mathrm{x}} / \mathrm{TiO}_{2}$ Powder. J. Phys. Chem C 2012, $116,23547-23555$.

(26) Sarode, P. R.; Priolkar, K. R. ; Bera, P.; Hegde, M. S.; Emura, V; Kumashiro, V. Study of Local Environment of $\mathrm{Ag}$ in $\mathrm{Ag} / \mathrm{CeO}_{2}$ Catalyst by EXAFS. Mater Res. Bull. 2002, 37, 1679-1690.

(27) Luches, P.; Pagliuca, F.; Valeri, S. Morphology, Stoichiometry, and Interface Structure of $\mathrm{CeO}_{2}$ Ultrathin Films on Pt(111). J. Phys. Chem. C 2011, 115, 10718-10726.

(28) Horcas, I.; Fernández, R.; Gómez-Rodríguez, J. M.; Colchero, J.; Gómez-Herrero, J.; Baro, A. M. WSXM: A Software for Scanning Probe Microscopy and a Tool for Nanotechnology. Rev. Sci. Instrum. 2007, 78, 013705. 
(29) Pascarelli, S.; Boscherini, F.; D'Acaptito, F.; Hrdy, J.; Meneghini, C.; Mobilio, S. X-ray Optics of a Dynamical Sagittal-Focusing Monochromator on the GILDA Beamline at the ESRF. $J$. Synchrotron Rad. 1996, 3, 147-155.

(30) Newville, M. IFEFFIT : Interactive XAFS Analysis and FEFF Fitting. J. Synchrotron Rad. 2001, 8, 322-324.

(31) Newville, M.; Ravel B. ATHENA, ARTEMIS, HEPHAESTUS: Data Analysis for X-Ray Absorption Spectroscopy Using IFEFFIT. J. Synchrotron Rad. 2005, 12, 537-541.

(32) Ankudinov, A.; Ravel, B.; Rehr, J.J.; Conradson, S. Real-Space Multiple-Scattering Calculation and Interpretation of X-Ray-Absorption Near-Edge Structure. Phys. Rev B, 1998, 58, 7565-7576.

(33) Luches, P.; Pagliuca, F.; Valeri, S. Structural and Morphological Modifications of Thermally Reduced Cerium Oxide Ultrathin Epitaxial Films on Pt(111). Phys. Chem. Chem. Phys. 2014, 16, 18848-18857.

(34) Tjeng, L. H. ; Meinders, M. B. J.; van Elp, J.; Ghijsen, J.; Sawatzky, G. A.; Johnson, R. L. Electronic structure of $\mathrm{Ag}_{2} \mathrm{O}$. Phys. Rev. B 1990, 41, 3190-3199.

(35) Bao, X.; Wild, U.; Muhler, M.; Pettinger, B.; Schlögl, R.; Ertl, G. Coadsorption of Nitric Oxide and Oxygen on the Ag(110) Surface. Surf. Sci. 1999, 425, 224-232.

(36) Zhou, J.; Baddorf, A. P.; Mullins, D. R.; Overbury, S. H. Growth and Characterization of Rh and Pd Nanoparticles on Oxidized and Reduced $\mathrm{CeO}_{\mathrm{x}}(111)$ Thin Films by Scanning Tunneling Microscopy. J. Phys. Chem. C 2008, 112, 9336-9345.

(37) Baron, M. ; Bondarchuk, O.; Stacchiola, D.; Shaikhutdinov, S.; Freund, H.-J. Interaction of Gold with Cerium Oxide Supports: $\mathrm{CeO}_{2}(111)$ Thin Films vs $\mathrm{CeO}_{\mathrm{x}}$ Nanoparticles. J. Phys. Chem. C 2009, 113, 6042-6049.

(38) Grinter, D. C.; Muryn, C.; Santos, B.; Shaw, B.-J.; Menteş, T. O.; Locatelli, A.; Thornton, G. Spectromicroscopy of a Model Water-Gas Shift Catalyst: Gold Nanoparticles Supported on Ceria. J. Phys. Chem C 2014, 118, 19194-19204.

(39) Wilson, E. L. ; Grau-Crespo, R.; Pang, C. L.; Cabailh, G.; Chen, Q.; Purton, J. A.; Catlow, C. R. A.; Brown, W. A.; de Leeuw, N. H.; Thornton, G. Redox Behavior of the Model Catalyst $\mathrm{Pd} / \mathrm{CeO}_{2-\mathrm{x}} / \mathrm{Pt}(111)$. J. Phys. Chem. C 2008, 112, 10918-10922.

(40) Højrup Hansen, K.; Worren, T.; Lægsgaard, E.; Besenbacher, F.; Stensgaard, I. Bias Dependent Apparent Height of an $\mathrm{Al}_{2} \mathrm{O}_{3}$ Thin Film on NiAl(110), and of Supported Pd Clusters. Surf. Sci. 2001, 475, 96-102.

(41) Napetschnig, E.; Schmid, M.; Varga, P. Pd, Co and Co-Pd Clusters on the Ordered Alumina Film on NiAl(110): Contact Angle, Surface Structure and Composition. Surf. Sci. 2007, 601, 32333245 . 
(42) Marks, L. D. Experimental Studies of Small Particle Structures. Rep. Prog. Phys. 1994, 57, 603-649.

(43) Cai, W. ; Zhong, H.; Zhang, L. Optical Measurements of Oxidation Behavior of Silver Nanometer Particle Within Pores of Silica Host. J. Appl. Phys. 1998, 83, 1705-1710.

(44) Schnippering, M.; Carrara, M.; Foelske, A.; Kötz, R.; Fermín, D. J. Electronic properties of Ag nanoparticle arrays. A Kelvin probe and high resolution XPS study Phys. Chem. Chem. Phys. 2007, 9, 725-730.

(45) Kern, R.; Müller, P. Elastic Relaxation of Coherent Epitaxial Deposits. Surf. Sci. 1997, 392, 103-133.

(46) Lagarde, P.; Colonna, S.; Flank, A.-M.; Jupille, J. Elastic Strains in Silver Clusters Supported on $\mathrm{MgO}(100)$. Surf. Sci. 2003, 524, 102-112.

(47) Mays, C. W.; Vermaak, J. S.; Kuhlmann-Wilsorf, D. On Surface Stress and Surface Tension: II. Determination of the Surface Stress of Gold. Surf. Sci. 1968, 12, 134-140.

(48) Montano, P. A.; Schulze, W.; Tesche, B.; Shenoy, G. K.; Morrison, T. I. Extended X-RayAbsorption Fine-Structure Study of Ag Particles Isolated in Solid Argon. Phys. Rev. B 1984, 30, 672677.

(49) Balerna, A.; Bernieri, E.; Picozzi, P.; Reale, A.; Santucci, S.; Burattini, E.; Mobilio, S. Extended X-Ray-Absorption Fine-Structure and Near-Edge-Structure Studies on Evaporated Small Clusters of Au. Phys. Rev. B 1985, 31, 5058-5065.

(50) Lin, C.-M.; Hung, T.-L.; Huang, Y.-H.; Wu, K.-T.; Tang, M.-T.; Lee, C.-H.; Chen, C. T.; Chen, Y. Y. Size-Dependent Lattice Structure of Palladium Studied by X-ray Absorption Spectroscopy. Phys. Rev. B 2007, 75, 125426.

(51) Apai, G.; Hamilton, J. F.; Stohr, J.; Thompson, A. Extended X-Ray-Absorption Fine Structure of Small $\mathrm{Cu}$ and Ni Clusters: Binding-Energy and Bond-Length Changes with Cluster Size. Phys. Rev. Lett. 1979, 43, 165-169.

(52) Sun, C. Q. Dominance of Broken Bonds and Nonbonding Electrons at the Nanoscale. Nanoscale 2010, 2, 1930-1961.

(53) Mulvaney, P. Surface Plasmon Spectroscopy of Nanosized Metal Particles. Langmuir 1996, $12,788-800$.

(54) Sancho-Parramon, J. Surface Plasmon Resonance Broadening of Metallic Particles in the Quasi-Static Approximation: a Numerical Study of Size Confinement and Interparticle Interaction Effects. Nanotechnology 2009, 20, 235706.

(55) Ng, K. H.; Liu, H.; Penner, R. M. Subnanometer Silver Clusters Exhibiting Unexpected Electrochemical Metastability on Graphite. Langmuir 2000, 16, 4016-4023. 
TOC graphic:

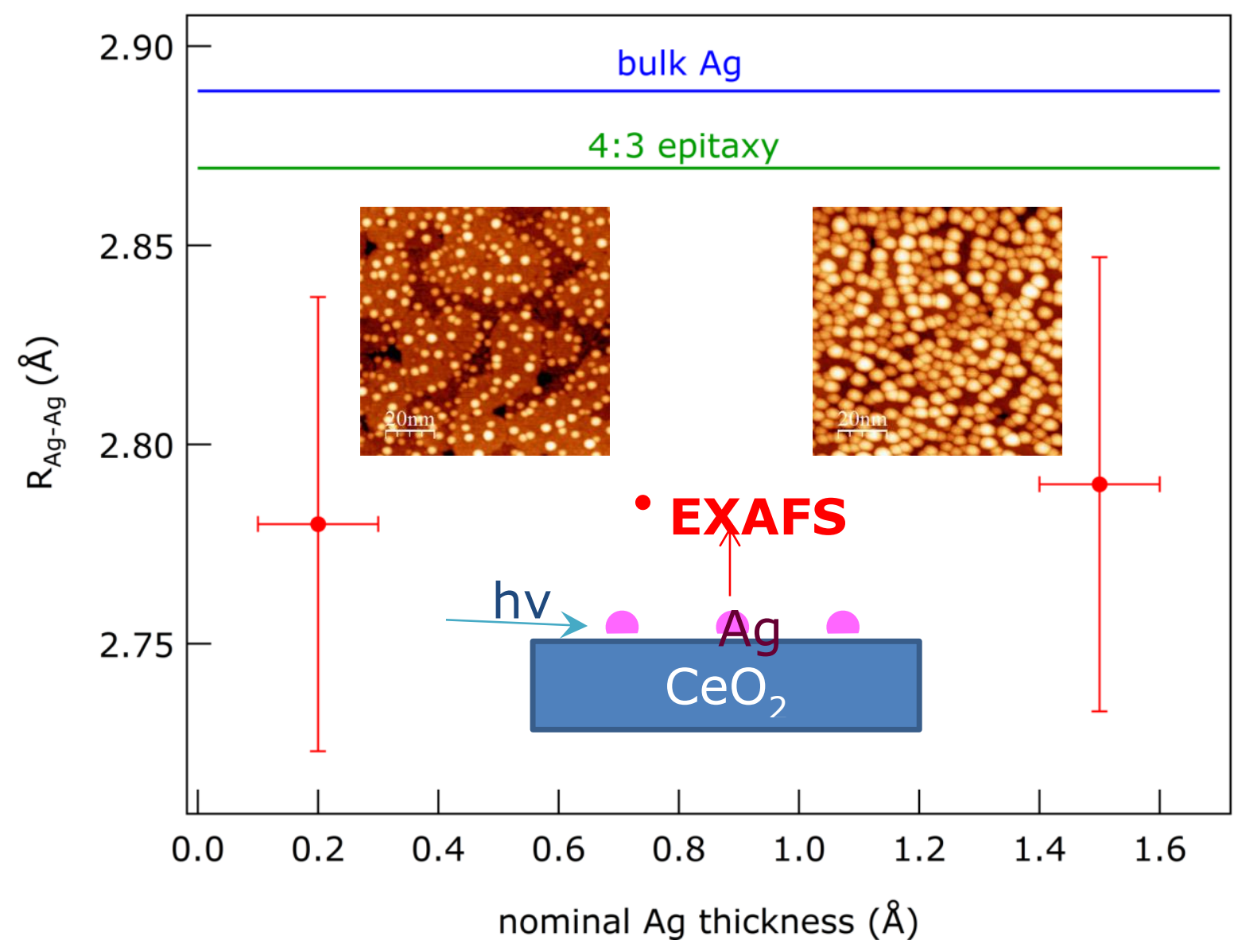

